\title{
Metal Nanowires Subjected to Relavant Hydrated Metal lons
}

\author{
Aiman Mukhtar, Tahir Mehmood* \\ Email address: \\ aleeza.mukhtar@yahoo.com (A. Mukhtar),tahir10621@yahoo.com (T. Mehmood) \\ ${ }^{*}$ Corresponding author
}

The State Key Laboratory of Refractories and Metallurgy, Hubei Collaborative Innovation Center for Advanced Steels, International Research Institute for Steel Technology, Wuhan University of Science and Technology, Wuhan, P. R. China

\section{To cite this article:}

Aiman Mukhtar, Tahir Mehmood. Metal Nanowires Subjected to Relavant Hydrated Metal Ions. International Journal of Materials Science and Applications. Vol. 6, No. 2, 2017, pp. 88-94. doi: 10.11648/j.ijmsa.20170602.14

Received: December 26, 2016; Accepted: January 24, 2017; Published: February 10, 2017

\begin{abstract}
The hcp-Co and fcc-Ni nanowires with the diameter of $\sim 50 \mathrm{~nm}$ were successfully prepared at different overpotentials by using potentiostatic electrochemical deposition in the pores of AAO templates. The prepared nanowires were characterized by X-ray diffraction, and the morphology of the nanowires was investigated by scanning electron microscope. In this study a new way is established to understand the growth rate of nanowires. The effect of work function on the growth rate of Co and Ni nanowires having same electrolyte's concentration, same $\mathrm{pH}$ value, and same overpotentials, has been discussed. The growth rate of metal nanowires in ECD is determined by the tunneling current between a metal and hydrated metal ions. The higher the tunneling current, the higher will be the growth rate. The tunneling current probably relates to the work function of metal. The larger is the value of work function the lower is the probability of electron tunneling. Lowering the work function causes increase in the current density. The hydrated Co and Ni ions are of octahedral structure with $\mathrm{M}-\mathrm{O}$ distance of $2.08 \AA$ and $2.05 \AA$, respectively. The work function of Co is smaller, this lead to higher tunneling current density. Therefore, the measured current density is higher for Co than for Ni and the Co nanowires grow faster than that of the Ni nanowires.
\end{abstract}

Keywords: Crystal Structure, Nucleation, Growth from Solutions, Deposition Parameters

\section{Introduction}

During the past decade, the potential utilization of nanostructured materials have promising research interest in the field of nanoelectric devices [1], high-density magnetic memories [2], high performance catalysts [3], and biomaterial separation membranes [4] and so on. Electrochemical methods are usually used for attaining thin films of numerous metals and semiconductors of conducting materials. Currently, the synthesis of nanowires by the electrochemical deposition is of great interest. To fabricate and investigate the growth mechanisms of metal nanowires in the cylindrical pores of a template is of essential and technological interests [5-10].

Bibliographical data present many synthesis methods of simple and multilayered nanowires such as: photochemical synthesis [11], catalytical synthesis [12], vapour-liquid-solid growing [13], lithographic patterning [14, 15] (are comparatively cumbrous, expensive and not appropriate for large scale fabrication), and template synthesis [16-20] via
Electrochemical Deposition (ECD) [21-23] in the pores of template. Template based technology is very useful in synthesizing one dimensional nano materials such as nanowires and nanotubes made of metals, semiconductors, polymers and carbon. Especially metal nanowires (Co, Ni, $\mathrm{Fe}, \mathrm{Ag}$, and $\mathrm{Cu}$ ) show great optical, electronic and magnetic properties. The template-assisted synthesis of nanowires is a conceptually simple and intuitive way to fabricate nanostructures $[24,25]$. These templates contain very small cylindrical pores or voids within the host material, and the empty spaces are filled with the chosen material, which adopts the pore morphology, to form nanowires. In templateassisted synthesis of nanostructures, the chemical stability and mechanical properties of the template, as well as the diameter, uniformity and density of the pores are important characteristics to consider. Template commonly used for nanowire synthesis is Anodic Aluminum Oxide (AAO) owing to its salient features such as decorative properties, nanopore symmetry and corrosion resistance $[18,20]$.

Highly ordered nanowires with uniform diameter, 
deposited on the AAO template, are essential to study their properties. Cobalt $(\mathrm{Co})$ and $\mathrm{Nickel}(\mathrm{Ni})$ nanowires have been electrochemically deposited into the pores of AAO template [26-29]. The earlier studies show that the growth surface of the fcc (Ni) [30, 31] and hep (Co) [32] metals is usually not only on the closely packed surface (atomically smooth surface) but also on the atomically rough surface. Our previous results show that deposition parameters have effect on structure of cobalt nanowires. Low electrolyte concentration $(0.35 \mathrm{M})$, deposition potential $(-1.6 \mathrm{~V})$ and high temperature $\left(60^{\circ} \mathrm{C}\right)$ favors the formation of hep Co nanowires and high electrolyte concentration (1.07M), deposition potential $(-3.0 \mathrm{~V})$ and room temperature $\left(25^{\circ} \mathrm{C}\right)$ favors the formation of fcc Co nanowires [33-35]. Recently T. Mehmood et al. discuss in his research article that the deposition rate of $\mathrm{Fe}$ nanowires are faster than $\mathrm{Co}$ and $\mathrm{Ni}$ nanowires because the current density of $\mathrm{Fe}$ is larger than $\mathrm{Co}$ and $\mathrm{Ni}$ nanowires at same $\mathrm{pH}$ of solution, Concentration and deposition voltage [36]. Little is known on the tunneling process occurring in the dehydration and also there is no other study for the effect of work function on the growth mechanism of $\mathrm{Co}$ and $\mathrm{Ni}$ nanowires. An effort has been made to explain the growth of these nanowires by considering the work function, using the electrochemical deposition method at different overpotentials.

\section{Experimental Detail}

Preparation of the AAO template: The porous anodic alumina oxide (AAO) templates were prepared via a two-step anodization procedure [30, 37, 38]. Prior to anodizing, highpurity aluminum foils (99.999\%) were first degreased in acetone and then were annealed in a vacuum of $10^{-5}$ Torr at $500{ }^{\circ} \mathrm{C}$ for $5 \mathrm{~h}$ to remove the mechanical stress, thus obtaining the aluminum foils with a homogeneous structure over a large area. The aluminum foils were first anodized in a $0.3 \mathrm{M}$ $\mathrm{H}_{2} \mathrm{C}_{2} \mathrm{O}_{4}$ (oxalic acid) solution at $2^{\circ} \mathrm{C}$ for $6 \mathrm{~h}$. After removing the alumina layer formed in the anodization in a mixture of phosphoric acid $(6 \mathrm{wt} \%)$ and chromic acid $(1.8 \mathrm{wt} \%)$, the aluminum foils were anodized again at the same conditions as the first step for $12 \mathrm{~h}$. The templates that experienced the above two-step anodization were etched in a saturated $\mathrm{CuCl}_{2}$ solution to remove the remaining aluminum on the back side. The alumina barrier layer was then dissolved in a $5 \mathrm{wt} \%$ phosphoric acid solution at $40^{\circ} \mathrm{C}$. In order to deposit metal into the pores of AAO templates, a gold film was sputtered onto the back side of the AAO templates to serve as the working electrode.

Electrodeposition of the metal nanowires: The potentiostatic ECD of the $\mathrm{Co}$ and $\mathrm{Ni}$ nanowires was performed in a conventional three electrode bath containing a mixture of $\mathrm{CoSO}_{4} .7 \mathrm{H}_{2} \mathrm{O}(0.356 \mathrm{M})$ and $\mathrm{H}_{3} \mathrm{BO}_{3}(0.68 \mathrm{M})$ solutions, and for the $\mathrm{Ni}$ nanowires mixture of $\mathrm{NiSO}_{4} \cdot 6 \mathrm{H}_{2} \mathrm{O}$ $(0.356 \mathrm{M})$ and $\mathrm{H}_{3} \mathrm{BO}_{3}(0.68 \mathrm{M})$ aqueous solutions. The $\mathrm{pH}$ value of the solution was adjusted to 2.5 with $1 \mathrm{M} \mathrm{H}_{2} \mathrm{SO}_{4}$. A copper plate and a Saturated Calomel Electrode (SCE) were used as counter electrode and reference electrode, respectively. The Co and Ni ECD was carried out at different deposition overpotentials $(\eta)(-0.9 \mathrm{~V}$, and $-0.6 \mathrm{~V}, \mathrm{SCE})$ at a room temperature (about $25^{\circ} \mathrm{C}$ ).

Characterization: The metal nanowires were characterized by X-ray Diffraction (XRD, Y-2000) with $\mathrm{CuK} \alpha$ radiations $(\lambda 0.154178 \mathrm{~nm})$. The images of deposited metal nanowires were obtained by Scanning Electron Microscope (SEM, JEOL JSM-6700F). For XRD measurements the film of $\mathrm{Au}$ was mechanically polished away. For SEM observations, the AAO templates were partly dissolved in $5 \mathrm{wt} \% \mathrm{NaOH}$ solution, and then carefully rinsed with deionized water for several times.

\section{Results and Discussion}

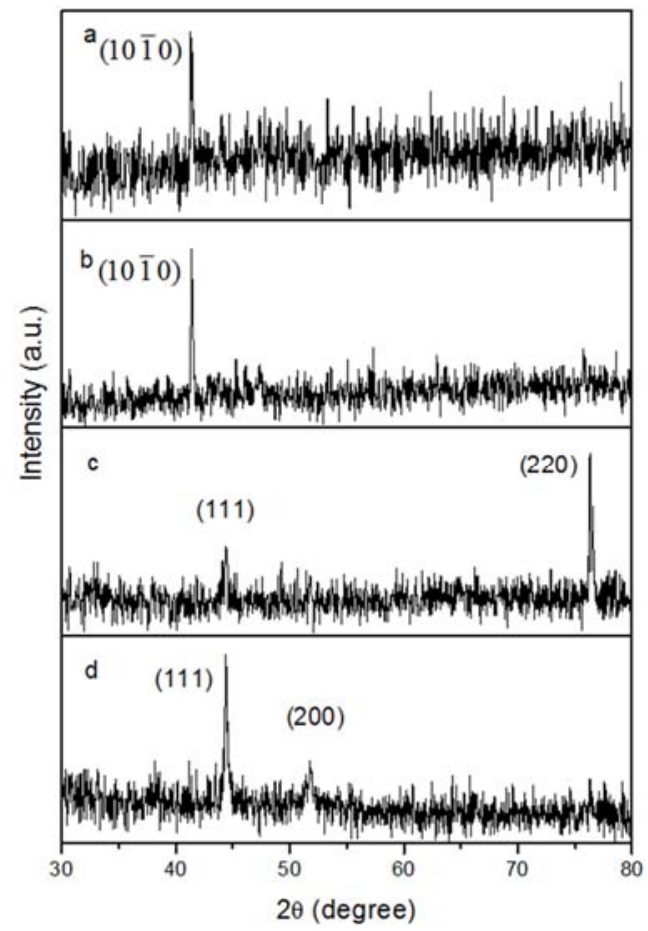

Figure 1. XRD pattern for electrochemically deposited nanowires in the pores of AAO template, a) Co at $\eta=-0.9 \mathrm{~V}$, b) Co at $\eta=-0.6 \mathrm{~V}$, c) Ni at $\eta=-$ $0.9 \mathrm{~V}$, and d) Ni at $\eta=-0.6 \mathrm{~V}$.

Figure 1 shows the XRD patterns of Co and Ni nanowires, which were collected from the top side of nanowires. The Co and $\mathrm{Ni}$ nanowires were prepared at a room temperature $\left(25^{\circ} \mathrm{C}\right)$ under the deposition overpotentials of $-0.9 \mathrm{~V}$, and $0.6 \mathrm{~V}$, SCE. The positions of the peaks are in good agreement with the indexed of Hexagonal closed pack (hcp) structure that is universal for electrodeposited Co and facecentered cubic (fcc) structure for Ni. All peaks match well with the (hcp) Co (JPCDS, 89-7094) and (fcc) Ni (JPCDS, 65-2865). Figure 1 (a, and b) illustrate the XRD patterns under $\eta=-0.9 \mathrm{~V}$ and $\eta=-0.6 \mathrm{~V}$ for Co nanowires, respectively. It can be seen from the figure there is only a single peak along $(10 \overline{1} 0)$ plane, which indicates well-preferred growth of Co nanowires along [10 $\overline{1} 0$ ] direction for both the potentials. Figure 1c represent the $\mathrm{Ni}$ nanowires at the potential of $-0.9 \mathrm{~V}$, it is clear from the figure that there are 
two peaks one along (220) plane and other along (111) plane, which means that some nanowires grow along [110] direction and others along [111] direction. However, the intensity for (220) planes is much higher than that of (111) planes, indicating that most nanowires still grow along the [110] direction. The XRD pattern for the Ni sample fabricated under the overpotential of $-0.6 \mathrm{~V}$ is shown in Figure $1 \mathrm{~d}$. With lowering the overpotential the peak intensity of (111) planes is much higher than that of (200) planes, presenting that most nanowires grow along [111] direction.

SEM usually produces images down to length scales of $\sim 10 \mathrm{~nm}$ and provides valuable information regarding the structural arrangement, spatial distribution, wire density, and geometrical features of the nanowires. Figure 2 gives a topview SEM image of AAO template, indicating that the pores on AAO template are hexagonally arranged and highly ordered. The average diameter and spacing of pores are estimated to be 50 and $100 \mathrm{~nm}$.

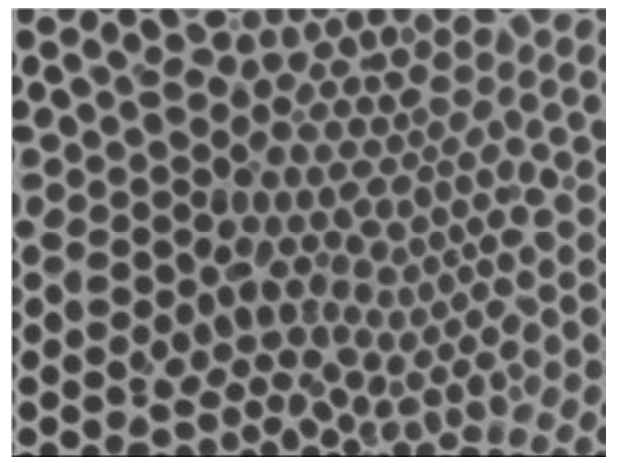

Figure 2. SEM images of the top view of highly ordered AAO template.
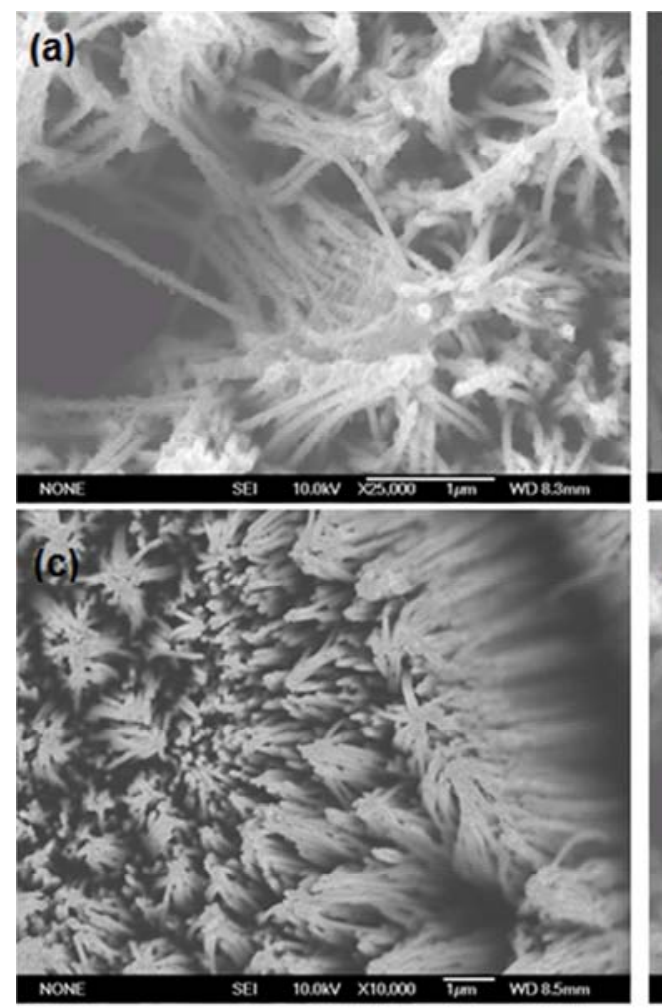

After the deposition of nanowires in pores, AAO templates were partly dissolved in $5 \mathrm{wt} \% \mathrm{NaOH}$ solution, and then carefully rinsed with deionized water for several times. The remains consisted of nanowires and a gold layer was served as a sample for SEM observation. Figure 3 shows SEM images of $\mathrm{Co}$ and $\mathrm{Ni}$ nanowires separated from the AAO templates. Figure 3 (a, and $b$ ) contain the SEM pictures for the Co nanowires at $\eta=-0.9 \mathrm{~V}$ and $\eta=-0.6 \mathrm{~V}$, respectively. The morphology of Ni nanowires obtained by SEM at $\eta=-0.9 \mathrm{~V}$ and $\eta=-0.6 \mathrm{~V}$ is illustrated in Figure 3 (c, and d). Diameter and length of the nanowires corresponds well to that of nanopores and the cylindrical shape was precisely transferred from the nanopores to the nanowires.

In order to follow the deposition process, the current density was recorded as a function of time. Figure 4 shows the current versus time curves for different overpotentials applied at $\mathrm{T}=25^{\circ} \mathrm{C}$. When an electric field is applied, the reduction of diffused cations is directly located towards cathode that result in the growth of nanowires inside the pores of template $[35,36]$.

Whitney et al., for the first time explained the mechanism of completely filling the nanopores of template during the film growth [6]. The abrupt fall of current take place when the potential is applied which shows that the nucleation starts at the pores bottom. S. Valizadeh et al., described that the distinctly decrease in current take place owing to the mass transport limitation [37, 38]. After that the current remains nearly unchanged till the complete filling of pores. At the end a sharp increase in current is observed due to over deposition on the template.

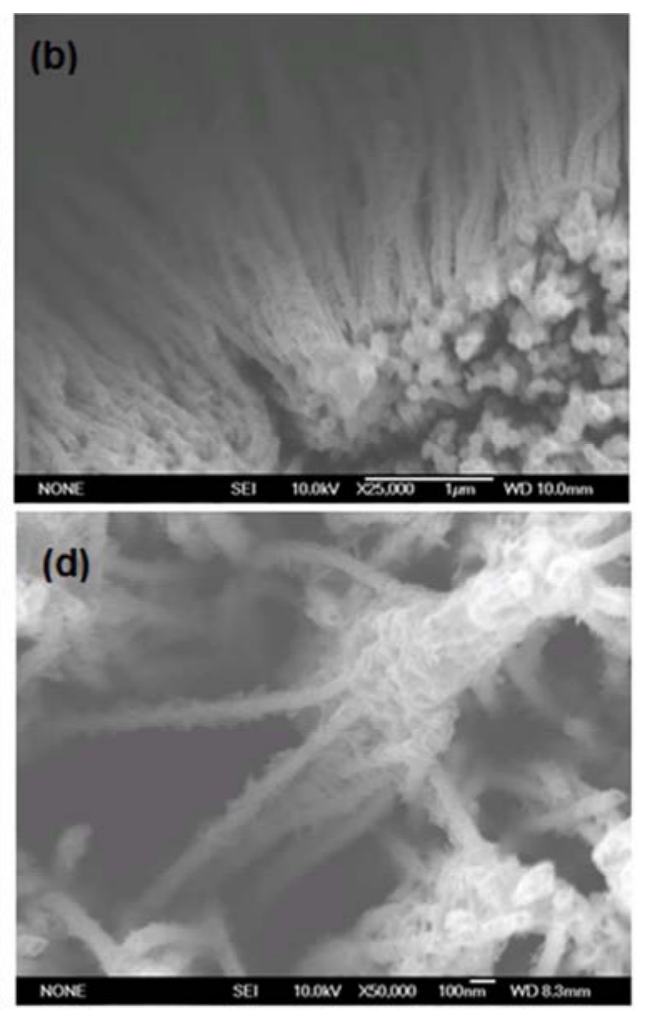

Figure 3. SEM pictures for $\mathrm{Co}(a, b)$ and $\mathrm{Ni}(c, d)$ at $\eta=-0.9 \mathrm{~V}$ and $\eta=-0.6 \mathrm{~V}$. 


\section{Discussion}

The electro-deposition is a complex process which includes charge transfer, diffusion, reaction, adsorption and substrate. Hence, the structure of metallic nanowires is closely linked to deposition conditions and growth modes during the process. Electrochemical processes are examined discussing to the ionic interactions at the electrodeelectrolyte interface. The metal receiving or gaining the electrons is the cathode since its cations in solution reduce or deposit on the cathode surface as atoms. Therefore, an electric field must exist in the electrolyte due to the presence of charge particles represented by ions [39]. At the metalelectrolyte interface a strong electric field exists and a double layer of charged particles interrupts the charge on the interface [40].

In electro-deposition of metal, a metal ion $\mathrm{M}^{\mathrm{n}+}$ is transferred from solution into the ionic metal lattice, meanwhile electrons are provided from the external electron source (power supply) to the electron gas of the metal M [41]. This electro-deposition process can be pictured as the following four steps in the atomic-level scale. First, hydrated metal ions in an aqueous solution diffuse to a metal surface and are adsorbed on this surface. (In a hydrated metal ion, the water molecules of hydration are electrostatically attached to the metal ion.) Second, when an adsorbed hydrated metal ion captures electrons from the surface by quantum-mechanical tunneling, the metal ion is neutralized. The electrostatic attractive interaction between the neutral metal atom and water molecules is zero and then the water molecules of hydration are displaced. Third, the neutral metal atom is adsorbed on the surface. Fourth, the adsorbed metal atom diffuses to a surface site (such as kink site) where it incorporates into the ionic metal lattice, thus leading to the growth on the surface on which the dehydration occurs. In the four steps described above, the second step plays a crucial role in the growth of metal nanowires. This is because the metal nanowires can grow only when the water molecules that are attached to the metal ions are displaced on a metal surface. The dehydration involves electron transfer from a metal surface to hydrated metal ions by tunneling [42].

The hydration reaction is defined as the transfer of an ion or a neutral chemical species from the gaseous phase into water; for metal ions $\mathrm{Mn}^{+}$(g) $\rightarrow \mathrm{Mn}^{+}$(aq). At this process, water molecules bind to metal ions through ion-dipole bonds of mainly electrostatic character and to anions through mainly hydrogen bonds. Tunneling processes take place in dehydration and few is known about these processes. In order to understand the tunneling processes in the dehydration, we first describe briefly the tunneling processes in field ion microscopy (FIM),[43] (See page 11 of this reference) which is well understood. In the presence of a high electrical field of a few $\mathrm{V} / \AA$, an electron in an atom near a metal surface can transfer to the metal surface by tunneling. In FIM, an electric field is generated at the surface when metallic needle is subjected to high voltage due to positive charges present at the surface. Due to strong electric field near the needle (tip) the occurring probability of field ionization is more. The width of the barrier is proportional to electric field, stronger the electric field higher will be the tunneling (this is because the width of tunneling barrier is reduced by the electrical field) [44]. When subjected to strong electric field, an electron from outer shell of gas atom tunnel through barrier towards the empty energy level at the metal surface. The ions are affected by the strong electric field near the tip. In FIM, the metal is applied a positive bias voltage, the electron of the image gas atom tunnels into the metal surface. In the case of electro-deposition, a metal surface is biased a negative voltage. The value of overpotential should be on the order of a few $\mathrm{V}$ with respect to adsorbed hydrated metal ions, which have a zero potential [45]. If the surface metal atom and adsorbed hydrated metal ion are regarded as the hard balls, the distance between the centers of them is several $\AA$. Therefore there is a high electrical field between the surface metal atom and adsorbed hydrated metal ion [46] (see page 5 of this reference). It is the high electrical field that leads to adsorption of hydrated metal ion on a metal surface and then electron tunneling from the metal surface to the adsorbed hydrated metal ion.

In a Scanning Tunneling Microscope (STM) the tunneling current decays exponentially (between tip and electrode). This tunneling current be influenced by the work function $(\phi)$ of metal and the distance (d) between the metal surface and hydrated metal ions, and can be expressed by the relations as,

$$
\mathrm{I}=\mathrm{A} \cdot \exp (-2 \mathrm{Kd})
$$

The tunneling decay constant $K$ can be defined operationally. One can further define an effective barrier height simply as

$$
K=\frac{\sqrt{2 m \varphi}}{\hbar}
$$

Where $\mathrm{m}$ is the mass of electron, $\phi$ is the work function of metal, $\mathrm{h}$ is the plank constant, and $\mathrm{d}$ is the distance between the metal surface and hydrated metal ions. Where $K$ is an experimentally measured quantity and is given by,

$$
K=-0.51 \sqrt{\varphi}
$$

The same expression of tunneling current in STM can be used in our work. The ratio of the tunneling current for two materials $\mathrm{Co}$ and $\mathrm{Ni}$ can be found by the following relation,

$$
I \mathrm{co} / I \mathrm{ni}=e 2 \times 0.51(-\sqrt{\varphi \mathrm{co}} \sqrt{\varphi n i})
$$

In this work, we tried to explain the growth mechanism of $\mathrm{Co}$ and $\mathrm{Ni}$ depending on the tunneling current. The distance between metal surface and hydrated metal ions is considered to be same for both the materials, but different at different overpotentials. The only factor we taken into account is the dependence of tunneling current on work functions of Co and 
Ni. It is seen that the tunneling current depends very sensitively on the work function of metal; the smaller is the $\phi$, the higher the tunneling current is.

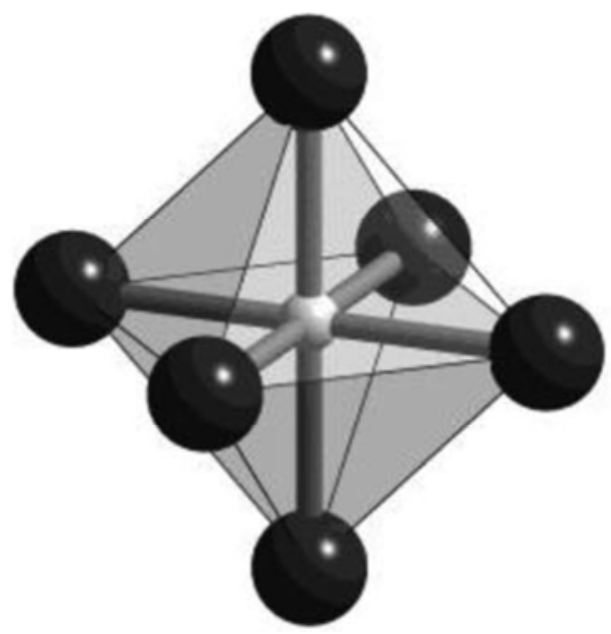

Figure 4. The octahedral structures of hydrated Co and Ni ions.

The $\mathrm{M}-\mathrm{O}$ bonds are mainly of electrostatic character, the coordination number of the hydrated metal ions is expected to be determined by the ratio of the ionic radius of the metal ion and the radius of the water oxygen atom [47]; the size of the water oxygen atom at coordination to metal ions has been determined to be $1.34 \AA$ [48]. The hydrated cobalt ion and the hydrated nickel ion are of octahedral configuration. The $\mathrm{M}-\mathrm{O}$ distance is $2.08 \AA$ for hydrated $\mathrm{Co}$ ion and $2.055 \AA$ for hydrated $\mathrm{Ni}$ ion with ionic radiuses of $0.74 \AA$ and $0.715 \AA$, respectively [48-51]. The value of work function of Co (5.0 $\mathrm{eV})$ is smaller than that of $\mathrm{Ni}(5.15 \mathrm{eV})$ [52]. When the three oxygen ion-formed $\mathrm{ABC}$ plane of hydrated $\mathrm{Co}$ and $\mathrm{Ni}$ ions contacts the metal surface, the distance between hydrated metal ion and the surface is shortest. Tunneling occurs with barriers of thickness around 10-30 $\AA$ and smaller [53]. That shortest distance between the metal surface and the hydrated metal ion at $\eta=-0.9 \mathrm{~V}$ is calculated to be $15 \AA$, and at $\eta=-0.6$ $\mathrm{V}$ is considered to be $18 \AA$. By using these values of $\mathrm{d}$ in the equation 2 we can get the ratio for current density at both the overpotentials. The ratio of current density for $\mathrm{Co}$ and $\mathrm{Ni}$ is 1.66 at $\eta=-0.9 \mathrm{~V}$, and 1.84 at $\eta=-0.6 \mathrm{~V}$. The aim is to find the effect of value of work function on the growth rate, for this, we considered the distance between metal surface and hydrated metal ions same for both Cobalt and Nickel.

The measured current density mainly consists of two parts. The first part $\mathrm{I}_{\mathrm{H}}$ is due to electron tunneling to $\mathrm{H}^{+}$ions. Since the $\mathrm{pH}$ value of the solutions for deposition of $\mathrm{Co}$ and $\mathrm{Ni}$ nanowires is the same, therefore, $I_{H}$ is the same during deposition of both Co and Ni nanowires. The second part $I_{M}$ is due to electron tunneling to hydrated metal ions. The $I_{M}$ for deposition of $\mathrm{Co}$ nanowires is different from that for deposition of $\mathrm{Ni}$ nanowires. As argued before tunneling current relies on the work function, lower the value of work function of metal higher will be the $\mathrm{I}_{\mathrm{M}}$, and so current density will be high. The work function of Co is smaller than that of the Ni metal leading to the higher tunneling current density.
Therefore, the measured current density is higher for Co than for $\mathrm{Ni}$ and the Co nanowires grow faster than that of the $\mathrm{Ni}$ nanowires.

\section{Conclusion}

In this study, Cobalt and Nickel nanowires were successfully electrochemically deposited into the nanopores of alumina templates. It is found that for the deposition of Co current density was higher than that of the Ni under the same conditions (mole concentration, overpotential, temperature, and $\mathrm{pH}$ ). This indicates fast growth rate of Co nanowires than that of Ni nanowires. The growth rate difference between $\mathrm{Co}$ and $\mathrm{Ni}$ can be explained by the tunneling current depending on the work functions of the materials. The equation used to explain the growth mechanism depends on work function and the distance between metal surfaces and hydrated metal ions. We tried to explain the tunneling current only depending on work function and considered the distance same for both Co and $\mathrm{Ni}$ for same overpotential. The lower is the work function higher is the tunneling current. The higher tunneling current density results faster growth rate, same phenomena is seen in our case. The work function for Co is $5.0 \mathrm{eV}$ and for $\mathrm{Ni}$ is $5.15 \mathrm{eV}$. The distances between the metal surface and hydrated metal ions are $15 \AA$ and $18 \AA$ at $\eta=-0.9 \mathrm{~V}$ and $\eta=-$ $0.6 \mathrm{~V}$, respectively for both the materials. The structure of Co and $\mathrm{Ni}$ ions is octahedral. As the value of work function for $\mathrm{Ni}$ is greater so the growth rate for the $\mathrm{Ni}$ is lower as compared to the Co.

\section{References}

[1] Gudiksen, M. S., et al., Growth of nanowire superlattice structures for nanoscale photonics and electronics. Nature, 2002. 415 (6872): p. 617-620.

[2] Sun, S., et al., Monodisperse FePt Nanoparticles and Ferromagnetic FePt Nanocrystal Superlattices. Science, 2000. 287 (5460): p. 1989-1992.

[3] Kim, S.-W., et al., Fabrication of Hollow Palladium Spheres and Their Successful Application to the Recyclable Heterogeneous Catalyst for Suzuki Coupling Reactions. Journal of the American Chemical Society, 2002. 124 (26): p. $7642-7643$.

[4] Lee, S. B., et al., Antibody-Based Bio-Nanotube Membranes for Enantiomeric Drug Separations. Science, 2002. 296 (5576): p. 2198-2200.

[5] Martin, C. R., Nanomaterials: A Membrane-Based Synthetic Approach. Science, 1994. 266 (5193): p. 1961-1966.

[6] Whitney, T. M., et al., Fabrication and Magnetic Properties of Arrays of Metallic Nanowires. Science, 1993. 261 (5126): p. 1316-1319.

[7] Wen-Ching Tsai, C.-C. W., and Yung-Yun Wang, Frequency Effect of Pulse Plating on the Uniformity of Copper Deposition in Plated Through Holes. J. Electrochem. Soc., 2003. 150 (5): p. 267-272. 
[8] A. J. Yin, J. L., W. Jian, A. J. Bennett, and J. M. Xu, Fabrication of highly ordered metallic nanowire arrays by electrodeposition. Applied Physics Letters, 2001. 79 (7): p. 1039 .

[9] El - Giar, E. M., et al., Localized Electrochemical Deposition of Copper Microstructures. Journal of The Electrochemical Society, 2000. 147 (2): p. 586-591.

[10] Routkevitch, D., et al., Electrochemical Fabrication of CdS Nanowire Arrays in Porous Anodic Aluminum Oxide Templates. The Journal of Physical Chemistry, 1996. 100 (33): p. 14037-14047.

[11] Kim, F. S., J. H. \& Yang, P. D, Photochemical synthesis of gold nanorods. Journal of American Chemical Society, 2002. 124 (48): p. 14316-14317.

[12] Huang, Y.D., X.F.; Cui, Y. \& Lieber, C. M, Gallium Nitride Nanowire Nanodevices. Nano Lettres, 2002. 2 (2): p. 101-104.

[13] Björk, M. T. O., B. J.; Sass, T.; Persson, A. I.; Thelander, C.; Magnusson M. H.; Deppert, K.; Wallenberg, L. R. \& Samuelson, L., One-dimensional Steeplechase for Electrons Realized. Nano Letters, 2002.2 (2): p. $87-89$.

[14] Wei Wu, B. C., Xiao-yun Sun, Wei Zhang, Lei Zhuang, Linshu Kong, and Stephen Y. Chou, Large area high density quantized magnetic disks fabricated using nanoimprint lithography. J. Vac. Sci. Technol. B, 1998. 16 (6): p. 3825-9.

[15] Gary M. McClelland, M. W. H., Charles T. Rettner, Margaret E. Best, Kenneth R. Carter, and Bruce D. Terris, Nanoscale patterning of magnetic islands by imprint lithography using a flexible mold. Appl. Phys. Lett., 2002. 81 (8): p. 1483-5.

[16] Whitney, T. M., et al., Fabrication and magnetic properties of arrays of metallic nanowires. Science (New York, N. Y.), 1993. 261 (5126): p. 1316-1319.

[17] J. M. García, A. A., J. Velázquez1, D. García, M. Vázquez, P. Aranda, and E. Ruiz-Hitzky, Magnetic behavior of an array of cobalt nanowires. J. Appl. Phys., 1999. 85 (8): p. 5480-2.

[18] D. J. Sellmyer, M. Z., R. Skomski, Magnetism of Fe, Co, and Ni Nanowires in Self-Assembled Arrays. J. Phys. Condens. Matter, 2001. 13: p. R433-R460.

[19] Skomski R, K. R. D. a. S. D. J., J. Appl. Phys., 1999. 85: p. 5069-71.

[20] Han G C, Zong B Y, and W. Y. H, IEEE Trans. Magn, 2002: p. $2562-4$

[21] Inguanta, R., S. Piazza, and C. Sunseri, Influence of the electrical parameters on the fabrication of copper nanowires into anodic alumina templates. Applied Surface Science, 2009. 255 (21): p. 8816-8823.

[22] Yu, Y. Y. C., S. S.; Lee, C. L. \& Wang, C. R. C., Gold Nanorods: Electrochemical Synthesis and Optical Properties. Journal of Physical Chemistry B, 1997. 101 (34): p. 66616664 .

[23] $\mathrm{Xu}, \mathrm{J}$. and K. Wang, Pulsed electrodeposition of monocrystalline $\mathrm{Ni}$ nanowire array and its magnetic properties. Applied Surface Science, 2008. 254 (20): p. 66236627.

[24] Ozin, G. A., Nanochemistry: Synthesis in diminishing dimensions. Advanced Materials, 1992. 4 (10): p. 612-649.
[25] Tonucci, R. J., Justus, B. J., Campillo, A. J., and Ford, C. E, NANOCHANNEL ARRAY GLASS. Science, 1992. 258 (5083): p. 783-785.

[26] K. Nielsch, R. B. W., J. Barthel, J. Kirschner, U. Gosele, S. F. Ficher, H. Kronmuller, Hexagonally ordered $100 \mathrm{~nm}$ period nickel nanowire arrays. Appl. Phys. Lett., 2001. 79 (9): p. $1360-2$.

[27] Fashen Li, T. W., Liyuan Ren and Jianrong Sun, Structure and magnetic properties of Co nanowires in self-assembled arrays. J. Phys.: Condens. Matter 2004. 16 (45): p. 8053.

[28] M. Darques, A. E., L. Vila, L. Piraux, Controlled changes in the microstructure and magnetic anisotropy in arrays of electrodeposited Co nanowires induced by the solution $\mathrm{pH}$. J. Phys. D: Appl. Phys., 2004. 37 (10): p. 1411.

[29] M. Darques, L. P., A. Encinas, P. Bayle-Guillemaud, A. Popa, U. Ebels, Electrochemical control and selection of the structural and magnetic properties of cobalt nanowires. Appl. Phys. Lett., 2005. 86 (7): p. 072508-72510.

[30] Wang XW, F. G., Xu XJ, Jin Z, Zhang LD, Size-dependent orientation growth of large-area ordered Ni nanowire arrays. J Phys Chem B., 2005. 109 (51): p. 24326-30.

[31] Xue Wei Wang, G. T. F., Li Chen, Xi Jin Xu and Li De Zhang, Orientation-Controllable Growth of Ni Nanowire Arrays with Different Diameters. Electrochem. Solid-State Lett., 2007. 10 (4): p. E1-E3.

[32] Huang, et al., Orientation-Controlled Synthesis and Ferromagnetism of Single Crystalline Co Nanowire Arrays. The Journal of Physical Chemistry C, 2008. 112 (5): p. 14681472.

[33] Mukhtar, A., et al., Effect of $\mathrm{Co} 2+$ concentration on the crystal structure of electrodeposited Co nanowires. Journal of Crystal Growth, 2016. 441: p. 26-32.

[34] Mehmood, T., et al., Mechanism for formation of fcc-cobalt nanowires in electrodeposition at ambient temperature. Materials Letters, 2014. 130 (0): p. 256-258.

[35] Mehmood, T., et al., Influence of bath temperature and $\mathrm{pH}$ on the structure of electrodeposited cobalt nanowires. International Journal of Materials Research, 2015. 106 (9): p. 957-961.

[36] Mehmood, T., et al., Growth Mechanism of Electrodeposited $\mathrm{Fe}, \mathrm{Co}$ and Ni Nanowires in the Form of Self-Assembled Arrays at Fixed Potential. Int. J. Electrochem. Sci, 2016. 11: p. 6423-6431.

[37] Biao Wang, G. T. F., Min Wang, Ming Guang Kong and Li De Zhang, Preparation of photonic crystals made of air pores in anodic alumina. Nanotechnology, 2007. 18 (36): p. 365601-4.

[38] Masuda, H. and K. Fukuda, Ordered Metal Nanohole Arrays Made by a Two-Step Replication of Honeycomb Structures of Anodic Alumina. Science, 1995. 268 (5216): p. 1466-1468.

[39] Levine, I. N., Physical Chemistry. McGraw-Hill Book Company, New York, 1978: p. 350-396.

[40] Jonghe, J. W. E. a. L. C. D., The Production of Inorganic Materials. Macmillan Publishing Company, New York, 1991: p. 1497-149.

[41] M. Paunovic and M. Schlesinger., Fundamentals of Electrochemical Deposition. 1998, New York: Wiley. 
[42] Persson, I., Hydrated metal ions in aqueous solution: How regular are their structures? Pure and Applied Chemistry, 2010. 82 (10): p. 1901.

[43] Tsong, T. T., Atom-probe field ion microscopy. 1990, Cambridge, New York: Cambridge University Press.

[44] Gomer, R., Field Emission and Field Ionisation Havard University, Cambridge, 1961.

[45] Paunovic, M. and M. Schlesinger, Fundamentals of Electrochemical Deposition.. 1998, New York: Wiley.

[46] Lower, S. K., Electrochemistry. Textbook: Simon Fraser University.

[47] Pearson, R. G., Hard and Soft Acids and Bases. Journal of the American Chemical Society, 1963. 85 (22): p. 3533-3539.

[48] Beattie, J. K., et al., Structural studies on the caesium alums, $\mathrm{CsM}\left[\mathrm{SO}_{4}\right.$ ] 2 [middle dot] $12 \mathrm{H}_{2} \mathrm{O}$. Journal of the Chemical Society, Dalton Transactions, 1981. 0 (10): p. 2105-2111.
[49] Ohtaki, H. and T. Radnai, Structure and dynamics of hydrated ions. Chemical Reviews, 1993. 93 (3): p. 1157-1204.

[50] Johansson, G., Structures of Complexes in Solution Derived from X-Ray Diffraction Measurements, in Advances in Inorganic Chemistry, A. G. Sykes, Editor. 1992, Academic Press. p. 159-232.

[51] Kristiansson, O., et al., A structural study of the hydrated and the dimethylsulfoxide, N, N'-dimethylpropyleneurea, acetonitrile, pyridine and $\mathrm{N}$, N-dimethylthioformamide solvated nickel (II) ion in solution and solid state. Inorganica Chimica Acta, 2003. 344: p. 15-27.

[52] Eastman, D. E., Photoelectric Work Functions of Transition, Rare-Earth, and Noble Metals. Physical Review B, 1970. 2 (1): p. 1-2.

[53] Trigg, L., Encyclopedia of Physics (second edition). New York, 1991: p. 1308. 